\title{
Breast Magnetic Resonance Imaging
}

\author{
Chair: Michael H. Fuchsjäger \\ Participants: Carla Boetes ${ }^{b} \quad$ Thomas Helbich $^{c}$ Sylvia H. Heywang-Köbrunner ${ }^{d}$ \\ Debra Ikeda $^{e}$ Elizabeth Morris ${ }^{f}$ Francesco Sardanelli $^{\mathrm{g}}$
}

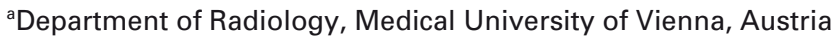

${ }^{b}$ Department of Radiology, Maastricht University Medical Center, The Netherlands

'Department of Radiology, Division Molecular and Gender Imaging, Medical University Vienna, Austria

${ }^{\mathrm{d}}$ Reference Center Mammography Munich and Breast Center Munich, Germany

eDepartment of Radiology, Stanford University, CA,

'Breast Imaging Service, Memorial Sloan-Kettering Cancer Center, New York, NY, USA

${ }^{9}$ Dipartimento di Scienze Medico-Chirurgiche, Servizio di Radiologia, Università degli Studi di Milano, Italy

\section{Question 1: What Are the Established Indications in which Breast MRI Represents the Current Gold Standard of Imaging?}

Boetes: Established indications for breast magnetic resonance imaging (MRI) are screening women with an increased risk for developing breast cancer; also in certain preoperative cases like invasive lobular carcinoma or in dense breasts or when there is a discrepancy in size on mammography and ultrasound, a breast MRI should be performed preoperatively.

Helbich: This has been adequately answered in a recent publication entitled: Magnetic resonance imaging of the breast: recommendations from the EUSOMA working group [Sardanelli et al., Eur J Cancer 2010;46:1296-316]. Briefly MRI is gold standard in the following situations: staging before treatment planning, screening of high-risk women, evaluation of response to adjuvant chemotherapy, patients with breast augmentation or reconstruction, search for occult breast cancer, exclusion of breast cancer recurrence.

Heywang-Köbrunner: MRI should be used in high-risk patients, in the search for an unknown primary (if conventional imaging is negative), to determine the status after breast conservation or reconstructive surgery with implant and suspicion of malignancy. Preoperative breast MRI should be considered in cases of lobular breast cancer or high-risk women unless the breast is easily assessed by conventional imaging.

Ikeda: i), Evaluating the effect of neoadjuvant chemotherapy on breast tumors before and after chemotherapy to establish tumor response (RECIST 1.1); ii), screening for breast cancer in women with a $20-25 \%$ lifetime risk for developing breast cancer and in women treated for Hodgkin's disease with informed consent [Saslow et al., CA Cancer J Clin 2007;57:7578]; iii) planning for partial breast irradiation (by showing cancer in a different quadrant in between $4-10 \%$ of cases that would not be treated by partial breast irradiation); iv) evaluating for a primary breast cancer in the breast that has an axillary lymph node containing metastases of breast cancer with negative other imaging; v) to evaluate the pectoralis major muscle invasion; vi) for MRI guided biopsies.

The indications for breast MRI that are still controversial have support in our evaluation for extent of disease in patients with newly diagnosed invasive lobular cancer, because MRI shows the extent of lobular cancer better than mammography or ultrasound. There is still controversy on whether breast MRI should be used to stage breast cancer in all patients due to the limitations of MRI. There is data that shows MRI does not decrease re-excision rates and that positive studies increase procedures for breast cancer patients. However, MRI does evaluate the contralateral breast for cancer, finding synchronous breast cancers in $3-4 \%$ of cases.

Morris: Breast MRI is currently the standard for high-risk screening. This is likely to be the most important application of breast MRI today. The challenge currently is to define who is at high risk for developing breast cancer and who could potentially benefit from this examination. Other applications include occult primary breast cancer, assessment of response to neoadjuvant chemotherapy, and assessment of positive margins following initial attempt at conservation therapy.

\section{KARGER}

Fax +497614520714

Information@Karger.de

www.karger.com (c) 2010 S. Karger GmbH, Freiburg

Accessible online at:

www.karger.com/brc
Ao. Univ.-Prof. Dr. Michael Fuchsjäger

Universitätsklinik für Radiodiagnostik

Medizinische Universität Wien, Allgemeines Krankenhaus Wien

Währinger Gürtel 18-20, 1090 Wien, Austria

Tel. +43 1 40400-4819, Fax -4898

michael.fuchsjaeger@meduniwien.ac.at 
Sardanelli: Established indications of MRI (i.e. indications largely agreed on by most breast cancer specialists) and MRI as a gold standard of imaging are the following: occult primary breast cancer; screening of high-risk women; suspected in-breast recurrence (when conventional imaging and needle biopsy are not conclusive); suspected rupture of a breast implant (unenhanced MRI sequences). MRI represents a gold standard but cannot be considered an established indication for the evaluation of the effect of neoadjuvant chemotherapy (NAC), i.e. MRI is not mandatory for all patients who undergo NAC. Debated indication: preoperative evaluation of ipsilateral and contralateral breast (the hottest point, see answer to question 2). A probable indication for the near future: nipple discharge.

\section{Question 2: What Are Today's Limitations of Breast MRI and How Do You Think They Will Be Overcome in the Near Future?}

Boetes: One of the limitations of breast MRI is the costs. At the moment it is much more expensive than conventional imaging and it is not always available. If breast MRI is performed, one should also have access to MRI guided interventions.

Helbich: MRI of the breast shows a rather high false positive rate. Multifunctional MRI of the breast including MR spectroscopy and diffusion weighted imaging, has the potential to significantly reduce this number. Of note, other imaging techniques like mammography or breast ultrasound are associated with a high false positive rate as well.

Heywang-Köbrunner: Its specificity is not bad, but it is too low for screening outside high-risk populations. Specificity drops with decreasing experience (the experience should not be measured by the number of MRI studies, but by the feedback on MRI positive or negative findings). Whenever a lesion is detected by MRI alone, histopathological assessment requires MR guided vacuum assisted breast biopsy or MR guided preoperative localization. These interventions increase the costs significantly (in most health care systems neither needles nor percutaneous biopsy are adequately or at all reimbursed). There is a lack of highly experienced teams who can provide this service. Furthermore, motion may be a source of error in the interpretation of some MRI studies, as it cannot be adequately avoided in some patients.

Ikeda: A major limitation of breast MRI is cost of the examination, its limited specificity and limitations and availability of MRI guided biopsy. Another limitation is the fund of knowledge of radiologists to interpret breast MRI. While the cost of breast MRI may decrease due to its increased availability, the cost of the contrast media needed to perform breast MRI will not decrease. As the experience of MRI interpreters increases, and as the scientific literature regarding breast MRI interpretation to increase its specificity increases, the specificity of MRI for breast cancer will go up.

Morris: With regard to staging, breast MRI may be a victim of its own success as it is capable of identifying multifocal and multicentric disease that may or may not be clinically relevant. It is well known that at the time of diagnosis most women with cancer have unsuspected multifocal or multicentric disease pathologically. Treatment algorithms have evolved to the point where most of this disease is treated either with radiation therapy or chemotherapy. It is almost as though the information on MRI that is currently available is a little too much too late. It seems paradoxical that once the cancer has been detected, surgical removal of every last focus of cancer may not be essential. This observation, however, could lead to non-surgical treatment of breast cancer. If the individual cancer lesions are small and therefore likely treated with radiation and chemotherapy then they might not need to be removed surgically. Another potential future application of MRI is to do 'less instead of more' in patients who may not require radiation therapy if no other areas of enhancement are identified within the breast.

However, it is likely that there are specific patient populations who would benefit markedly from the use of breast MRI in the preoperative setting. Unfortunately, these subpopulations have not been extensively examined. In my opinion, women with dense breasts and young women are the patients who would benefit most from preoperative breast MRI. This limitation (of detecting very small cancers) with respect to staging, however, is an advantage when talking about screening and that is why screening with breast MRI is likely to take off.

Sardanelli: The main limitation is not related to the technique itself. In fact, we lack well-designed and well conducted large randomized controlled trials (RCTs) demonstrating not only superior diagnostic performance of breast MRI, but a significant advantage in terms of outcome. In particular, this is true for the preoperative and neaodjuvant settings. Moreover, the recent results of the COMICE trial [Turnbull et al., Lancet 2010;375:563-71] showed a not significant reduction of the re-operation rate for positive margins for the MRI arm. The study has relevant limitations [Sardanelli, Breast Cancer Res Treat 2010;124:717-21], but this remains an open issue. A real intrinsic limitation of breast MRI is the need of intravenous injection of contrast agent (with the only exception of implant evaluation). There is an increasing interest for non-contrast breast MRI approaches - diffusion-weighted imaging (DWI) and MR spectroscopy (MRS) - but it is too early to think about non-contrast breast MRI as a routine clinical tool. At any rate, DWI is the best candidate for this purpose. 


\section{Question 3: According to Literature, MRI Has the Highest Sensitivity for Detection of Breast Cancer. However, the Low Diagnostic Specificity May Lead to False Positive Results. What Are Your Strategies to Address this Issue?}

Boetes: To increase the specificity high temporal sequences should be added to sequences with a high spatial resolution. There might also be a role for diffusion imaging.

Helbich: It is correct that MRI of the breast particularly in inexperienced hands may lead to a high false positive rate. Training and a standardized technique can significantly reduce this limitation. On the other hand, a positive finding does no indicate malignancy. It only says that this specific finding needs further assessment, most likely with MR guided needle biopsy. MR guided needle biopsy was shown to be an alternative to open breast biopsy with a very low false negative rate (less than $2 \%$ ).

Heywang-Köbrunner: We try to schedule according to the menstrual cycle and ask to stop hormone replacement therapy with progesterone for at least 4 weeks. There is no breast MRI without exact correlation with conventional imaging. We limit the indications to patients with sufficient risk.

Ikeda: Strategies to increase specificity include high spatial and temporal resolution, the use of $\mathrm{T} 2$-weighted imaging to evaluate for fluid or cells; knowing the pre-test probability of whether or not the patient may have cancer will also increase specificity.

Morris: The clinical context is very important to me when interpreting a breast MRI. I have become less concerned of foci when interpreting a staging examination, assuming the patient will receive standard treatment. Additionally, on highrisk screening examinations I am very hesitant to biopsy a finding on an initial screening examination without a comparison study unless it is very suspicious. I no longer follow initial screening examinations for suspected background parenchymal enhancement. Background parenchymal enhancement is likely responsible for many of the false positive findings and understanding and recognition of this entity likely will result in fewer biopsies.

Sardanelli: First of all, I would like to highlight that breast MRI does not have a low specificity. The meta-analysis by Peters et al. published in Radiology in 2008 [Radiology 2008;246:116-24] showed that breast MRI specificity is $72 \%$, surely comparable if not higher than that of mammography or ultrasound. In the screening setting of high-risk women, specificity is as high as $97 \%$ with a positive predictive value (PPV) of $56 \%$ (the HIBCRT-1 study, Sardanelli et al., Invest Radiol, in press). How to manage a PPV of 50-60\%, not significantly different from that of mammography and ultrasound? With second look targeted ultrasound (which detects from $60-80 \%$ of findings initially detected only with MRI) and ultrasound guided biopsy; with MR guided biopsy for the remaining cases. DWI and MRS can be useful for managing difficult special cases (i.e. cases not reachable with MR guidance).

\section{Question 4: How Did the Advent of 3-Tesla Magnets, Diffusion and Spectroscopy MRI Techniques Change Our Diagnostic Approach and Performance? Should these Technical Developments Be Used in Any or Just in Academic Settings?}

Boetes: To my opinion especially MRI spectroscopy should be used in selected cases like in the evaluation of neoadjuvant chemotherapy. 3-Tesla machines will be used in the near future not only in academic settings but also in public hospitals.

Helbich: Multifunctional 3 Tesla MRI of the breast including high spatial, high temporal, contrast enhanced MRI, diffusion weighted imaging and MR spectroscopy has the potential to offer a strong imaging tool to reduce the false positive rate significantly. This multifunctional approach offers the possibility to securely use MRI of the breast as an imaging tool in the differentiation of benign and malignant breast lesions. These technical developments are of importance; however, they are not ready to be launched in a clinical setting. Further studies are necessary to work on standardized protocols and quantitative analysis approaches.

Heywang-Köbrunner: Our experiences with diffusion have been disappointing. MR spectroscopy may be of interest for the assessment of neoadjuvant chemotherapy. So far more prospective studies are needed.

Ikeda: New technologies such as diffusion weighted imaging, or MR spectroscopy have shown promise, but have not shown definitively increased specificity for breast cancer; further investigation is still needed. Across the world, however, the use of 3-Tesla magnets, with their increased ability to generate fast, high-resolution images has already overtaken the marketplace in the United States. Although there is no publication showing marked penetrance of the 3-Tesla magnets into the marketplace, market forces will undoubtedly drive most facilities to acquire 3-Tesla magnets.

Morris: Unfortunately, these applications do not at the time of this writing have widespread clinical use. I hold the most hope for diffusion weighted imaging as this sequence is easy to perform and fast. In the clinical setting, this is a feasible add-on sequence or stand-alone sequence possibly in the future. It is possible that diffusion weighted imaging could be 
a fast non-contrast screening option in the future. Spectroscopy yields a wealth of information, however, it is time-consuming laborious and not currently automated. If multi-voxel techniques could be devised and the examination could be performed quickly, this technique could have clinical use.

Sardanelli: The advantages of 3-Tesla magnets do not seem to be so relevant if compared with an updated 1.5-Tesla magnet used with an 8-channel coil, even though the higher spectral resolution could be useful for MR spectroscopy. MR spectroscopy is a fantastic tool for tumor metabolic study and may be used for a very early evaluation of the NAC effect. We have done a lot of investigational work on this and we recently published on breast lesion characterization with MR spectroscopy [Sardanelli et al., AJR Am J Roentgenol 2009;192: 1608-17], but I don't think MR spectroscopy can be used as a routine clinical tool. Conversely, DWI shows a huge potential, as recently demonstrated by Whoodams et al. in the NAC setting [Radiology 2010;254:357-66]. DWI can be used in all hospitals or medical centers.

\section{Question 5: Where Do We Currently Stand with MRI Guided Minimal Invasive Therapy Approaches for Localized Breast Cancer? What Will Be Valid Indications in the Future for this Modality to Serve as an Alternative/Adjunct for Established Treatment Options?}

Boetes: MRI guided minimal invasive therapy is at the moment something just used in academic settings. I don't know if this will become an established treatment option. A lumpectomy with or without reconstruction can also give a good cosmetic outcome.

Helbich: I do see great potentials for MR guided focused ultrasound. The first dedicated units are already on the market and promising results have been reported. I don't want to say that MRI guided focused ultrasound can immediately replace open surgery; however, this tool should not be underestimated.

Heywang-Köbrunner: To date insufficient data exist concerning the use of MRI guided therapy for localized breast cancer. Overall, MRI has the advantage of being able to visualize temperature distribution. First investigations mainly concerning MRI guided focused ultrasound are encouraging.

Ikeda: MRI guided minimally invasive therapy approaches are still investigational. In the United States, we still depend on pathology to determine whether or not the margin of a surgical specimen is positive. Until MRI cannot definitively show the extent of breast cancer, undoubtedly we will still do excisional biopsies for breast cancer treatment. This is because the surgeon and radiation oncologist determine whether the surgical margins are positive to determine if the patient may undergo locally applied radiation therapy such as accelerated partial breast irradiation, or whether they need whole breast radiation.

Morris: Most definitely I think that MRI invasive therapy has an application of the future. As the understanding of breast cancer has evolved, it has become evident that the basic biology of breast cancer is important and that not all breast cancers are the same. Using targeted therapy along with tumor ablation for larger lesions may become standard in the future. Targeted therapy for small lesions may be all that is required. Different tumor biology types may also require different treatment approaches.

Sardanelli: Minimally invasive therapy (MIT) of localized breast cancer is the future of breast cancer treatment as an alternative to open surgery. In my view, this is the continuation of the trend of reduced treatment initiated by the Italian school of Umberto Veronesi more than 30 years ago and today reinforced by the practice of partial breast irradiation, recently authorized in the U.S. also outside research. Less is always better, if the patient outcome is good. MIT is also the only way to face the problem of overdiagnosis and overtreatment related to screening mammography. There is a big debate on the amount of overdiagnosis with estimates ranging from $<10 \%$ to $30 \%$ and higher. However, a certain rate of overdiagnosis cannot be avoided in whatever screening program. To reduce the aggressiveness of treatment is the only exit strategy. MRI is the best candidate to guide targeted energy for treating small breast cancers, using focused ultrasound, radiofrequency ablation or cryoablation. But all these are investigational tools. We are in the early beginning. Here again we need well designed studies, possibly randomized clinical trials.

\section{Question 6: With Regard to the Intermediate Future (5 Years from Now), Will Breast MRI Be an Indispensable Stand-Alone Modality for Diagnostic Breast Imaging or Screening of the General Population (without the Need for Mammography or Ultrasound)?}

Boetes: I am convinced that in the near future MRI will become a stand-alone method to evaluate the breasts. We have to see that the results of conventional screening are quite disappointing, the number of breast cancers in the western countries is increasing and the results of MRI screening in women with an increased risk for breast cancer are very good.

Helbich: Had anyone thought that MRI of the breast has the potential to replace mammography and ultrasound in highrisk patients 5 years ago? Maybe not in 5 years, but there is 
the potential that MRI of the breast replaces mammography screening. However, this is also a question of costs and in this case, mammography has been unbeatable until recently.

Heywang-Köbrunner: No, unless novel information becomes available.

Ikeda: Current data indicate that breast MRI still misses up to $25 \%$ of ductal carcinomas in situ (DCIS), which is shown by mammography alone as indicated by the presence of microcalcifications. For this reason MRI should not be the standalone breast imaging screening tool of the general population. Furthermore, breast MRI is too expensive and difficult to maintain compared to X-ray mammography. However, if breast MRI can be shown to demonstrate DCIS, MRI will be the screening tool of choice.

Morris: Improvement in techniques which allow assessment of the breast parenchyma possibly without the use of contrast agents, such as diffusion weighted imaging, may widen the application of breast MRI to screen the population at large. This would only be feasible if the false positive rate is low. Additionally, MRI specific contrast agents which could be targeted to cancer cells may also allow more specific imaging and could possibly localize the sites of cancer within the breast and elsewhere within the body.

Sardanelli: This is probable for high-risk women (BRCA1 or BRCA2 mutation carriers, etc.); because there is no added diagnostic value of mammography and/or first look ultrasound in a high-risk woman who had a negative breast MRI. Both the German study (the EVA trial [Kuhl et al., J Clin Oncol 2010;28:1450-7]) and the Italian study (HIBCRIT-1) clearly show this. However, it's not probable at all for the general female population. Take into consideration that clinical practice evolves much more slowly than technological development. Also, screening practice evolves more slowly. Before introducing MRI into screening we should demonstrate that MRI is better than mammography for screening women at intermediate risk (i.e. those women without familiar predisposition who were previously treated for a breast cancer), not only in terms of diagnostic performance but in terms of patient outcome. Here again, RCTs are needed.

\section{Participants}

Prof. Dr. Carla Boetes

Department of Radiology

Maastricht University Medical Center (MUMC)

P.O. Box 5800, 6202 AZ Maastricht, The Netherlands

Tel. + 31 43-3876910, Fax. -2876909

c.boetes@mumc.nl

Thomas Helbich, MD, MSc, MBA

Department of Radiology

Division: Molecular and Gender Imaging

Medical University Vienna - General Hospital Vienna

Währinger Gürtel 18-20, Floor 7F, 1090 Vienna, Austria

Tel. +43 1 40400-4819, Fax -4898

thomas.helbich@meduniwien.ac.at

Prof. Dr. med. Sylvia H. Heywang-Köbrunner

Reference Center Mammography Munich and Breast Center Munich, Einsteinstr. 3, 81675 München

Tel. +49 89 961661-330

heywangkoe@referenzzentrum-muenchen.de

Debra M. Ikeda, M.D.

Department of Radiology

Stanford University

875 Blake Wilbur Drive, Stanford, CA 94305-5826, USA

Tel. +1 650 723-8462, Fax -7562

dikeda@stanford.edu

Elizabeth A. Morris, MD, FACR

Breast Imaging Service

Memorial Sloan-Kettering Cancer Center

1275 York Avenue New York, NY 10065, USA

Tel. +1 646 888-4510, Fax: -4010

morrise@mskcc.org

Prof. Francesco Sardanelli

Università degli Studi di Milano,

Dipartimento di Scienze Medico-Chirurgiche,

Direttore del Servizio di Radiologia,

IRCCS Policlinico San Donato,

Via Morandi 30, 20097 San Donato Milanese, Milan, Italy.

francesco.sardanelli@unimi.it 